\title{
Science MIAAAS
}

Planetary Radio Astronomy Observations from Voyager 1 near Saturn Author(s): J. W. Warwick, J. B. Pearce, D. R. Evans, T. D. Carr, J. J. Schauble, J. K. Alexander, M. L. Kaiser, M. D. Desch, M. Pedersen, A. Lecacheux, G. Daigne, A. Boischot, C. H. Barrow

Source: Science, New Series, Vol. 212, No. 4491 (Apr. 10, 1981), pp. 239-243

Published by: American Association for the Advancement of Science

Stable URL: http://www.jstor.org/stable/1685671

Accessed: 09/12/2010 15:26

Your use of the JSTOR archive indicates your acceptance of JSTOR's Terms and Conditions of Use, available at http://www.jstor.org/page/info/about/policies/terms.jsp. JSTOR's Terms and Conditions of Use provides, in part, that unless you have obtained prior permission, you may not download an entire issue of a journal or multiple copies of articles, and you may use content in the JSTOR archive only for your personal, non-commercial use.

Please contact the publisher regarding any further use of this work. Publisher contact information may be obtained at http://www.jstor.org/action/showPublisher?publisherCode=aaas.

Each copy of any part of a JSTOR transmission must contain the same copyright notice that appears on the screen or printed page of such transmission.

JSTOR is a not-for-profit service that helps scholars, researchers, and students discover, use, and build upon a wide range of content in a trusted digital archive. We use information technology and tools to increase productivity and facilitate new forms of scholarship. For more information about JSTOR, please contact support@ jstor.org. 
Fig. 8. The radio emission intensity at 56.2 $\mathrm{kHz}$ as a function of the subsolar SLS longitude and the Dione orbital phase angle.

by which this distortion could affect the radio emission is not clear.

Although Saturn's rotation has a major effect on the radio emission process, other factors are also involved since the intensity of the events varies substantially on time scales of days. Figure 7 shows a 16-day plot of the $56.2-\mathrm{kHz}$ intensities centered on closest approach. In addition to the basic rotational control an overall modulation of the intensities can be seen with a period slightly less than 3 days. For instance, intense events are present on days $313,316,318$ and 319 , 321 , and 324. Because of the well-known control of Jovian radio emissions by the moon Io, an obvious explanation of this periodicity is that one of the moons of Saturn is influencing its radio emissions. Dione is the only moon with a suitable period, 2.74 days. The orbital phase angle of Dione is shown in the bottom panel of Fig. 7. The correlation appears to be quite good. A further analysis using all of the available data shows the radio emission intensity at $56.2 \mathrm{kHz}$ as a function of both the subsolar SLS longitude and the Dione orbital phase (Fig. 8). The significance of the combined control by these two parameters is best seen by comparing the relative intensities in the upper left and lower right quadrants. The largest radio emission intensities occur when the subsolar SLS longitude is near $90^{\circ}$ and Dione is passing through the local morning region of the magnetosphere. The best correlation with Dione's position occurs during the few weeks around closest approach, when the intensities are largest.

The apparent control of the Saturnian radio emission intensities by Dione suggests that this moon may be involved in a strong magnetospheric interaction, possibly resembling the interaction of Io with the Jovian magnetosphere. Most likely this interaction would imply outgassing and production of plasma by Dione, similar to the situation at Io. Although there is no evidence of volcanic activity, the photographs of Dione show white wispy features indicating the loss of volatile material (15). Furthermore, the Pioneer 11 plasma measurements show a peak in the plasma density profile near Dione's orbit (7), suggesting that Dione is a source of plasma. If Dione is injecting plasma into the magnetosphere, numerous mechanisms could account for the dependence on orbital position, including magnetospheric

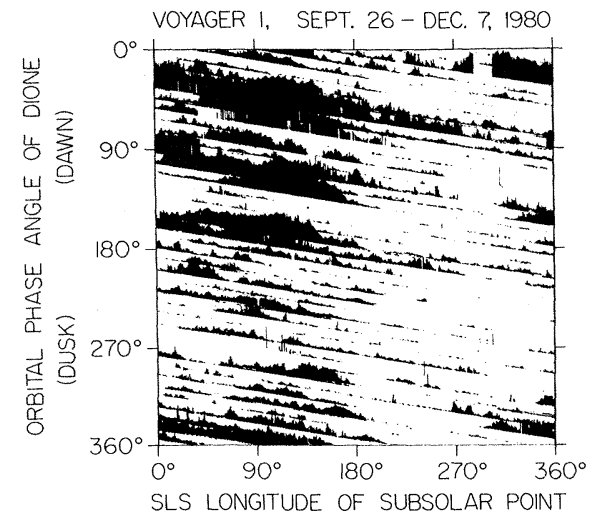

dawn-dusk asymmetries, propagation effects due to Dione-induced density variations in the plasma torus, and phase variations in the outgassing rate due to surface asymmetries on Dione.

D. A. GurnetT

W. S. KURTH

Department of Physics and Astronomy, University of Iowa, Iowa City 52242

F. L. SCARF

Space Sciences Department, TRW

Defense and Space Systems Group.

Redondo Beach, California 90278

References and Notes

1. F. L. Scarf and D. A. Gurnett, Space Sci. Rev.

2. 21, 289 (1977). W. S. Kurth, Science 204, 991 (1979); D. A. Gurnett, et al., ibid. 206, 987 (1979).

3. P. Rodriguez, J. Geophys. Res. 84, 917 (1979); F. L. Scarf, W. W. L. Taylor, C. T. Russell, R.

4. N. F. Ness, M. H. Acuña, R. P. Lepping, J. E. P. Connerney, K. W. Behannon, L. F. Burlaga, F. M. Neubauer, Science 212, 211 (1981).

\section{Planetary Radio Astronomy Observations from}

\section{Voyager 1 Near Saturn}

Abstract. The Voyager 1 planetary radio astronomy experiment detected two distinct kinds of radio emissions from Saturn. The first, Saturn kilometric radiation, is strongly polarized, bursty, tightly correlated with Saturn's rotation, and exhibits complex dynamic spectral features somewhat reminiscent of those in Jupiter's radio emission. It appears in radio frequencies below about 1.2 megahertz. The second kind of radio emission, Saturn electrostatic discharge, is unpolarized, extremely impulsive, loosely correlated with Saturn's rotation, and very broadband, appearing throughout the observing range of the experiment (20.4 kilohertz to 40.2 megahertz). Its sources appear to lie in the planetary rings.

After the discovery of radio emissions from Saturn in January $1980(1)$, the Voyager 1 planetary radio astronomy (PRA) experiment continued to record nonthermal radio emissions from the planet throughout the year. The data are the basis for our determination of a fairly precise rotation period representative of the internal convective regions of Saturn which generate the planetary magnetic field (2).

This report discusses the major results obtained with the PRA equipment (3) during the Voyager 1 flight past Saturn in November 1980 . The results fall into two categories, one the product of the expanded knowledge we have gained of the Saturn low-frequency emissions, and the other an unexpected and apparently unique broadband, impulsive radiation that appeared in our records within a few days of closest approach on 12 November.

Saturn kilometric radiation. Saturn is 


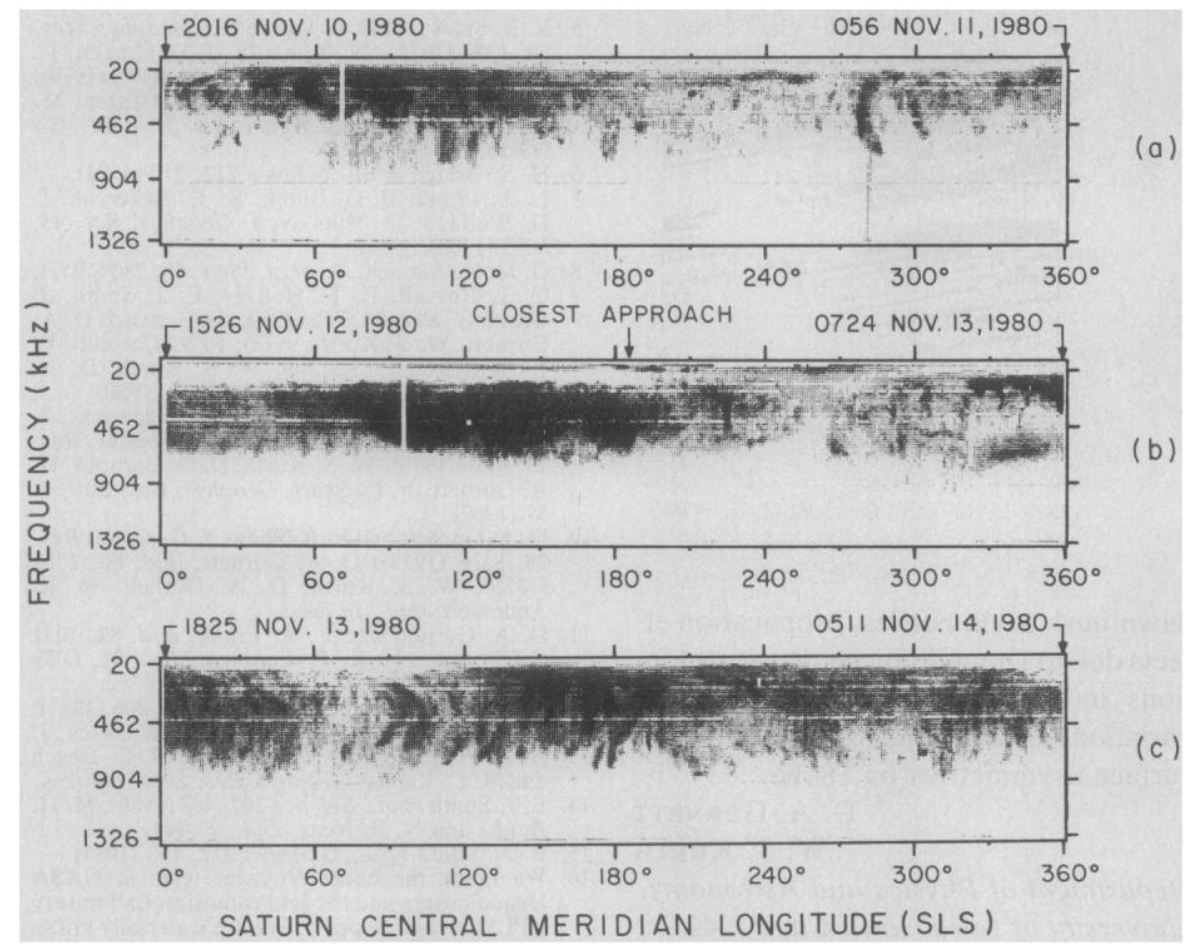

Fig. 1. Dynamic spectra of SKR for three complete rotations of the planet. In each plot the darkness of the shading is proportional to signal intensity as a function of wave frequency and SLS. (a) Data for a 10.657-hour interval 2 days before closest approach to Saturn; (b) covers a full $360^{\circ}$ SLS centered on closest approach and (c) shows spectra 1 day after closest approach. A type III solar radio burst appears in (a) at $281^{\circ}$ SLS, beginning at the highest frequencies and drifting rapidly into the stronger Saturn emission at frequencies near $700 \mathrm{kHz}$.

so strong a radio source at kilometer wavelengths that we detected Saturn kilometric radiation (SKR) when Voyager was still more than 3 astronomical units (AU) from Saturn. During the Saturn encounter period the very large increase in the sensitivity of the PRA observations allowed us to examine the SKR in detail.

Figure 1 shows three representative samples of SKR activity. The signal intensity at Voyager 1 is displayed in shades of gray as a function of frequency and time. Each panel corresponds to one complete rotation of the planet or a full $360^{\circ}$ range of longitude at Saturn's central meridian (2). We see a broad band of emission extending from the lowest PRA frequency channel $(20 \mathrm{kHz})$ to $\sim 1 \mathrm{MHz}$. The maximum intensity is usually observed at frequencies between about 100 and $500 \mathrm{kHz}$, and the half-power bandwidth is typically 200 to $400 \mathrm{kHz}$. The high-frequency cutoff is particularly steep; no SKR has been detected above $1200 \mathrm{kHz}$. A typical value for the isotropic equivalent total radiated power is $10^{8}$ to $10^{9} \mathrm{~W}$, but on some occasions we have observed power levels that exceed this value by more than an order of magnitude. Although there appears to be some observable emission at all times, there are distinct variations in intensity levels on a time scale of a few hours and on much shorter scales. At times the emis- sion seems to be organized into relatively narrowband structures that drift systematically in frequency as a function of time. Note the "open parenthesis" feature at about $280^{\circ}$ SLS in Fig la and the multiple "close parentheses" between about $90^{\circ}$ and $120^{\circ}$ SLS in Fig. 1c. Such distinct, dynamic spectral features are

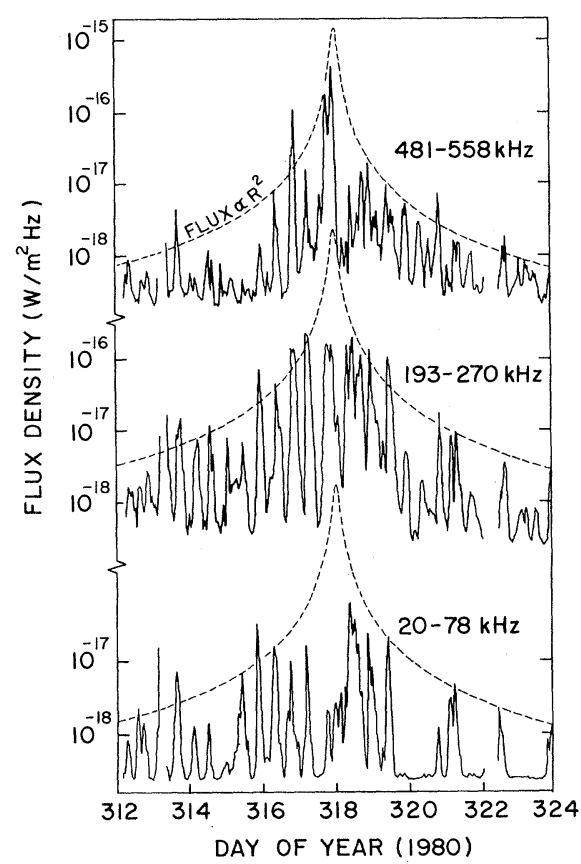

Fig. 2. Hourly averages of power flux density. A very strong modulation pattern occurs at the 10.657-rotation period of the planet. reminiscent of the arcs and drifting structures commonly seen in Jupiter's radio emissions (4).

Occasionally the SKR appears to be divided into several separate frequency bands. For example, in Fig. 1b between $90^{\circ}$ and $210^{\circ}$ SLS there is a narrow band of emission confined to frequencies below about $100 \mathrm{kHz}$. In the range above $100 \mathrm{kHz}$, the SKR often tends to split into two bands-one centered near 200 $\mathrm{kHz}$ and a second at or above $400 \mathrm{kHz}$. For example, note the interval between $60^{\circ}$ and $120^{\circ}$ in Fig la and the interval between $300^{\circ}$ and $360^{\circ}$ in Fig. 1b. We do not yet know whether there are three distinct SKR components.

In Fig. 2 are plotted hourly averages of the received power flux density, as measured by the PRA receiver in three separate frequency bands during the 12-day period centered on closest approach. At all frequencies there is a strong periodic modulation of the emission due to the rotation of the planet (rotation period, 10.657 hours). The amplitude of the modulation is sometimes more than two orders of magnitude, suggesting that the radiation is very sharply beamed or that some aspect of the magnetospheric environment that corotates with the planet exerts a strong control over the times when SKR is generated. The intensity levels are variable on time scales longer than the rotation period, so there may be considerable differences in the flux density at a given frequency from one rotation to the next. There is evidence, at frequencies up to $\sim 200 \mathrm{kHz}$, of regular long-period modulation of the SKR intensity levels. For example, in the bottom curve in Fig. 2 the emission has relative minima at 2 - to 3 -day intervals centered at days $312.0,314.6,317.5$, 320.1, 322.0, and 323.2. Power spectral analysis of the interval from days 309 through 328 confirms that, in addition to the 10.657-hour rotation modulation, there is modulation at $\sim 65$ hours (5). Within measurement accuracy, this corresponds to Dione's revolution period about Saturn. This 65-hour modulation is particularly apparent for the interval within a few days of closest approach, but seems to diminish as the data span is increased. Analysis of more than 9 months of SKR cruise data shows no significant modulation at this period.

The dashed curves in Fig. 2 show how the flux density from a steady source at Saturn should vary during the encounter if it follows the dependence of intensity on distance $(R)$ stated by the inversesquare law. The curves have been arbitrarily placed to fit the flux density maxima observed away from the time of closest approach. The $R^{-2}$ trend matches 
the data reasonably well except for the 1to 2-day period near closest approach, where the flux densities fall short of the smooth curve by more than $10 \mathrm{~dB}$. The flux density shortfall (compared to the $R^{-2}$ prediction) is most pronounced in the two lower frequency bands. This effect occurred as the encounter trajectory took Voyager 1 into the southern hemisphere down to latitudes below $40^{\circ} \mathrm{S}$. The data therefore suggest at least 10 -dB asymmetry between sources in the northern and southern hemispheres.

The PRA instrument operates as a polarimeter sensitive to circular or elliptical polarization. During closest approach, the angle between the line of sight to Saturn and the plane containing the PRA antennas came close to 90 ; at this angle the polarimeter array is optimally oriented to measure the true axial ratio of the SKR waves. The signals in the two polarization channels were then different by more than $20 \mathrm{~dB}$-close to the nominal performance limit of the PRA receiver electronics. It indicates that the wave axial ratio of SKR is $>80$ percent and corresponds to nearly pure circularly polarized waves.

During the long series of observations of SKR in the first half of 1980 , all events were right-hand ( $\mathrm{RH})$-polarized. Beginning in early September 1980, left-hand (LH)-polarized emission was detected. The number of LH-polarized bursts steadily increased with time, and nearly equal levels of $\mathrm{RH}$ - and LH-polarized signals were detected as the spacecraft came within 1 Saturn radius $\left(R_{\mathrm{S}}\right)$ of the equatorial plane. When Voyager was $\sim 1 R_{\mathrm{S}}$ south of the equatorial plane the polarization became $\mathrm{LH}$ and remained so until Voyager was again $\pm 1 R_{\mathrm{S}}$ from the equatorial plane (northbound). The polarization then again became mixed. When Voyager was $\sim 1 R_{\mathrm{S}}$ north of the equatorial plane the polarization became exclusively RH. The situation we have summarized applies to the frequency range near and below the peak of the SKR spectrum $(\leq 500 \mathrm{kHz})$. At higher frequencies the polarization did not become LH until the spacecraft was well below the equatorial plane. The other exception to the pattern of polarization reversal at southern latitudes was in the case of narrowband low-frequency components $(\leq 80 \mathrm{kHz})$, which remained RH-polarized throughout the near-encounter period.

The simplest interpretation of the polarization reversal at negative latitudes is a southern hemisphere source. That is, southern hemisphere extraordinarymode emission propagating away from the planet is LH-polarized and northern hemisphere emission is $\mathrm{RH}$-polarized.

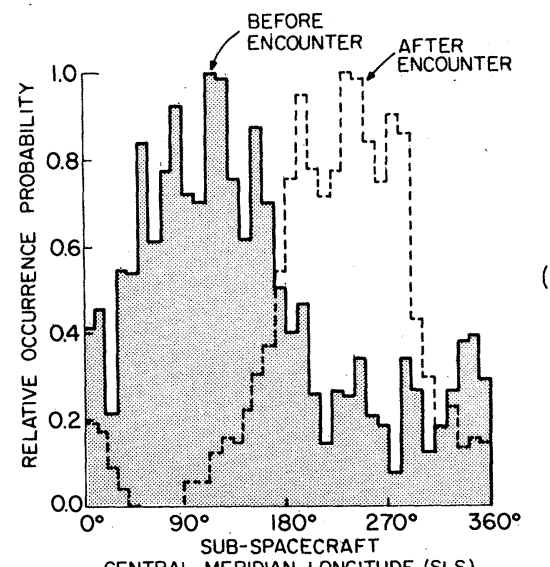

CENTRAL MERIDIAN LONGITUDE (SLS)

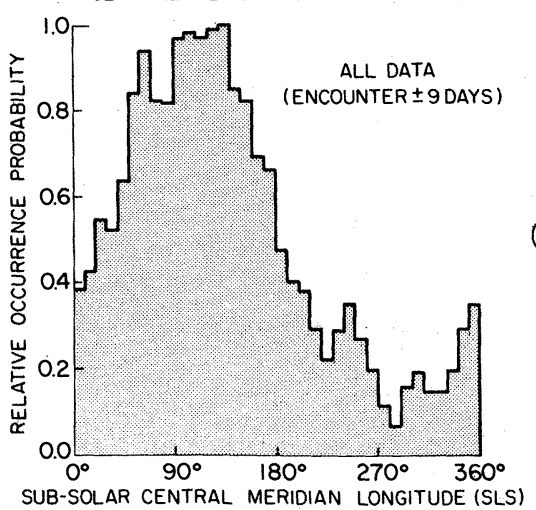

SUB-SOLAR CENTRAL MERIDIAN LONGITUDE (SLS)

During the approach to Saturn at a latitude of $8^{\circ} \mathrm{N}$, Voyager was detecting primarily northern hemisphere RH emission. The occasional weak LH events correspond to southern hemisphere emission. After closest approach, the spacecraft latitude increased to $25^{\circ} \mathrm{N}$. The LH southern hemisphere events have not been observed at the high postencounter northern latitudes.

As we noted in connection with Fig. 2, the principal modulation of SKR is due
Fig. 3. (a) Occurrence probability of SKR as a function of SLS, showing both pre- and postencounter distributions. (b) Occurrence probability of SKR for the entire encounter period (4 to 23 November 1980), plotted as a function of the subsolar SLS. Each histogram shows the relative occurrence of activity at $174 \mathrm{kHz}$ at power flux densities $>10^{-20} \mathrm{~W} \mathrm{~m}^{-2} \mathrm{~Hz}^{-1}$ when normalized to a distance of $1 \mathrm{AU}$ between Saturn and the observer.

to the rotation of the planet. Earlier we presented evidence that the detection probability is a maximum near $90^{\circ}$ SLS (1). As is apparent in Fig. 3a, this is not the case after encounter: the preferred longitude for detection of SKR from the northern hemisphere shifted by nearly $135^{\circ}$ toward higher longitudes. During the encounter the spacecraft moved prograde around Saturn through a total Saturnocentric angle of $\sim 225^{\circ}$ with respect to the sun. For the present discussion the SKR shift of $135^{\circ}$ in SLS is a retrograde angle that can be expressed as $225^{\circ}$ in the prograde sense. It is about the same sense and magnitude as the change in the Saturn local time of the spacecraft between the pre- and postencounter geometries. This result clearly indicates a source that may not rotate with the planet and that emits only when a preferred Saturn longitude coincides with a certain local time meridian (Fig. 3b).

Saturn electrostatic discharges. To us, the biggest surprise of the Voyager 1 encounter with Saturn appeared only during the days immediately preceding, during, and after closest approach. Figure 4 shows typical dynamic spectra at closest approach. These are created by 6-second step scans at $30 \mathrm{msec}$ per step in $128200-\mathrm{kHz}$-wide channels uniformly spaced from 40.2 to $1.2 \mathrm{MHz}$ and 701 -
Fig. 4. Dynamic spectral scans near closest approach. The raster scan of frequencies lies parallel to the frequency scale. The SED phenomenon appears as short streaks parallel to the frequency scale. The duration of the discharges is proportional to the height of the streaks, the overall height being almost exactly 6 seconds. The streaks are distributed quite randomly, from $20 \mathrm{kHz}$ to $40 \mathrm{MHz}$, and last $\sim 100 \mathrm{msec}$

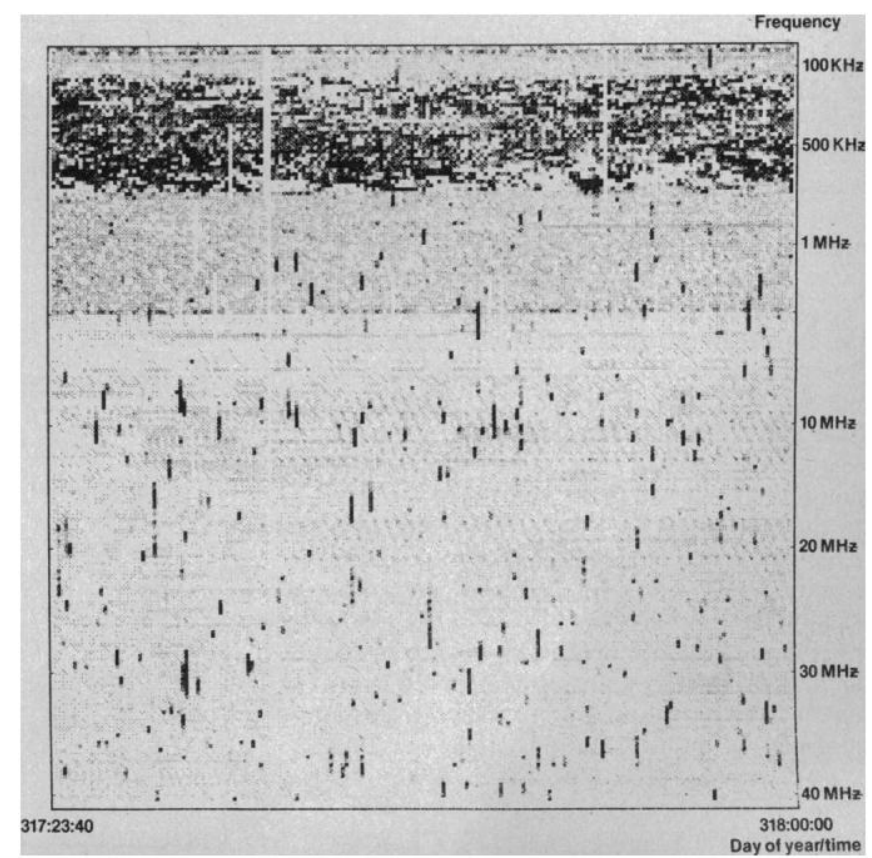




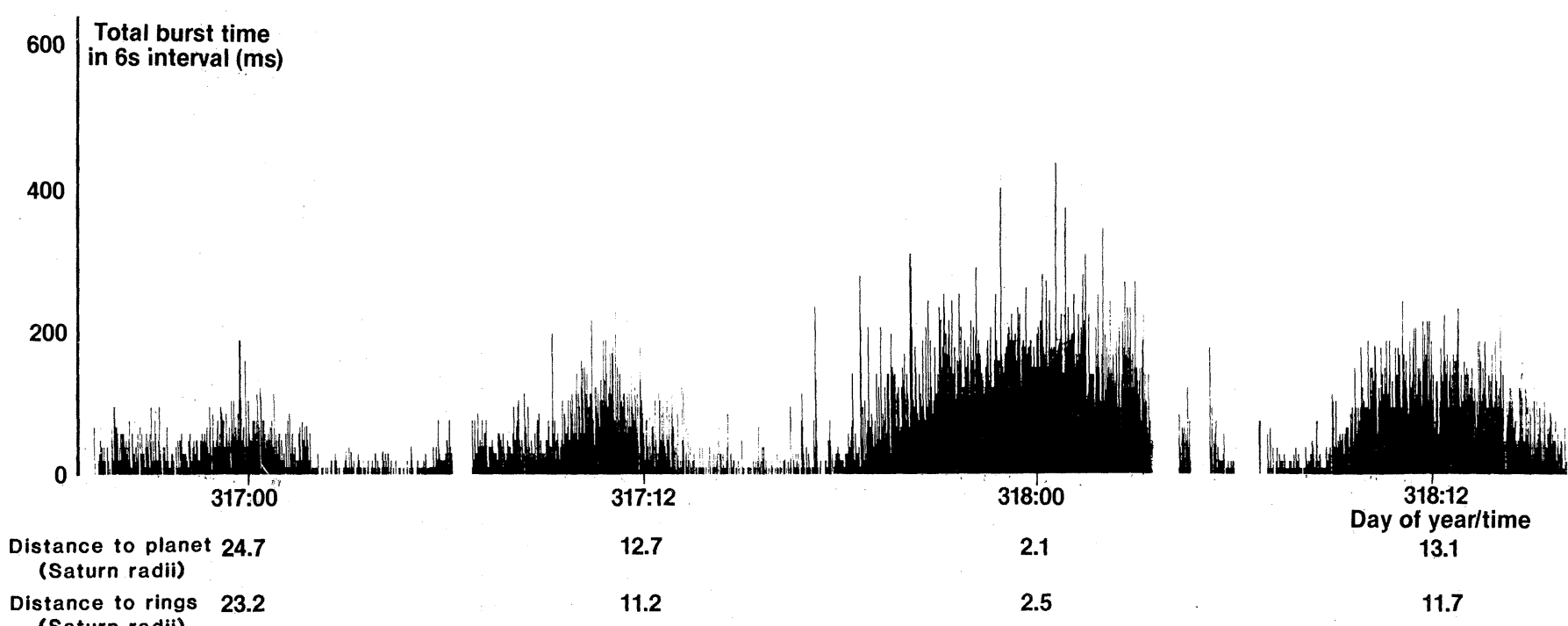

Fig. 5. Occurrence of SED as a function of time through closest approach, which occurs near 318:00.

$\mathrm{kHz}$-wide channels uniformly spaced from $1.326 \mathrm{MHz}$ to $20.4 \mathrm{kHz}$. SKR appears in the frequency range 100 to 700 $\mathrm{kHz}$. In addition, at all frequencies short vertical streaks appear, each confined to a single 6-second scan. Note that in Fig. 1 , which fails to show the streaks, there is an integration over 48 seconds and a normalization designed to exhibit details at peak intensity.

The spacecraft and its various subsystems apparently were operating normally during the interval covered in Fig. 4 and during our gathering of virtually all similar data. The possibility of poor data quality seems to be ruled out by (i) the measured quality of the telemetry link, (ii) the rate of occurrence of the pulses compared to the nominal bit error rate, and (iii) the nonrandom occurrence of the pulses, which all display increases in the same bit position in several consecutive eight-bit telemetry bytes, each data

Fig. 6. High-rate data in channels at 9.5 and $9.8 \mathrm{MHz}\left(14.4 \times 10^{3}\right.$ samples per second and $200-\mathrm{kHz}$ bandwidth) near closest approach. The two frequencies are sampled simultaneously at intervals of about $140 \mu \mathrm{sec}$. The channel polarizations lie in the two orthogonal circular states. The SED occur simultaneously on the two frequencies and their appearance is identical in the two polarization states. The expanded time scale shows a single SED. This single event is thrice folded; each point represents a sample in the $\mathrm{RH}$ polarization state and is spaced $140 \mu \mathrm{sec}$ from its neighbors. This isolated discharge shows rapid fluctuations over 20 to $30 \mathrm{~dB}$ of received flux. Its fluctuations are essentialy constant; that is, its variability does not appear to define the beginning, end, or middle in any special way. Finally, to simplify the display, the points representing the $\mathrm{LH}$ polarization state are not displayed. The LH points are observed simultaneously with the RH points and so do not improve the time resolution. However, there is no tendency of the points to "pair off"; even on this fine time scale, the SED is unpolarized. sample consisting of one byte. Furthermore, the impulsive discharges are independent of the telemetry mode of the spacecraft. Such independence would be highly unlikely were the discharges generated by a defect in the telemetry.

Conditions near Saturn may have affected the spacecraft in such a way as to create discharges near it, on it, or in it. It was possible to examine many hours of PRA high-rate (115.2 kilobits per second) data from Jupiter for the presence of electrostatic discharges. A few were discovered, with time constants of the order of $\leq 100 \mu \mathrm{sec}$. No Jupiter events lasting the equivalent of even one dwell time (30 msec) were observed. If the discharges at Saturn were generated near the spacecraft through deleterious environmental phenomena, such as are known to produce electrostatic discharges on terres- trial spacecraft, why were the discharges not seen at Jupiter? This argument supports the view that the source of the discharges was external to the spacecraft.

Another possibility is that these were discharges at the spacecraft but that they were generated through a different mechanism than that underlying the classical spacecraft electrostatic discharge. Saturn's environment presumably is filled with a hierarchy of dielectric particles of all sizes. If they were charged, they might generate electrical events as they strike the PRA antenna booms. The time scale of the discharge against the PRA booms would be only a fraction of a nanosecond, however-much faster than we observe.

There were some $10^{4}$ Saturn electrostatic discharges (SED) during the 48
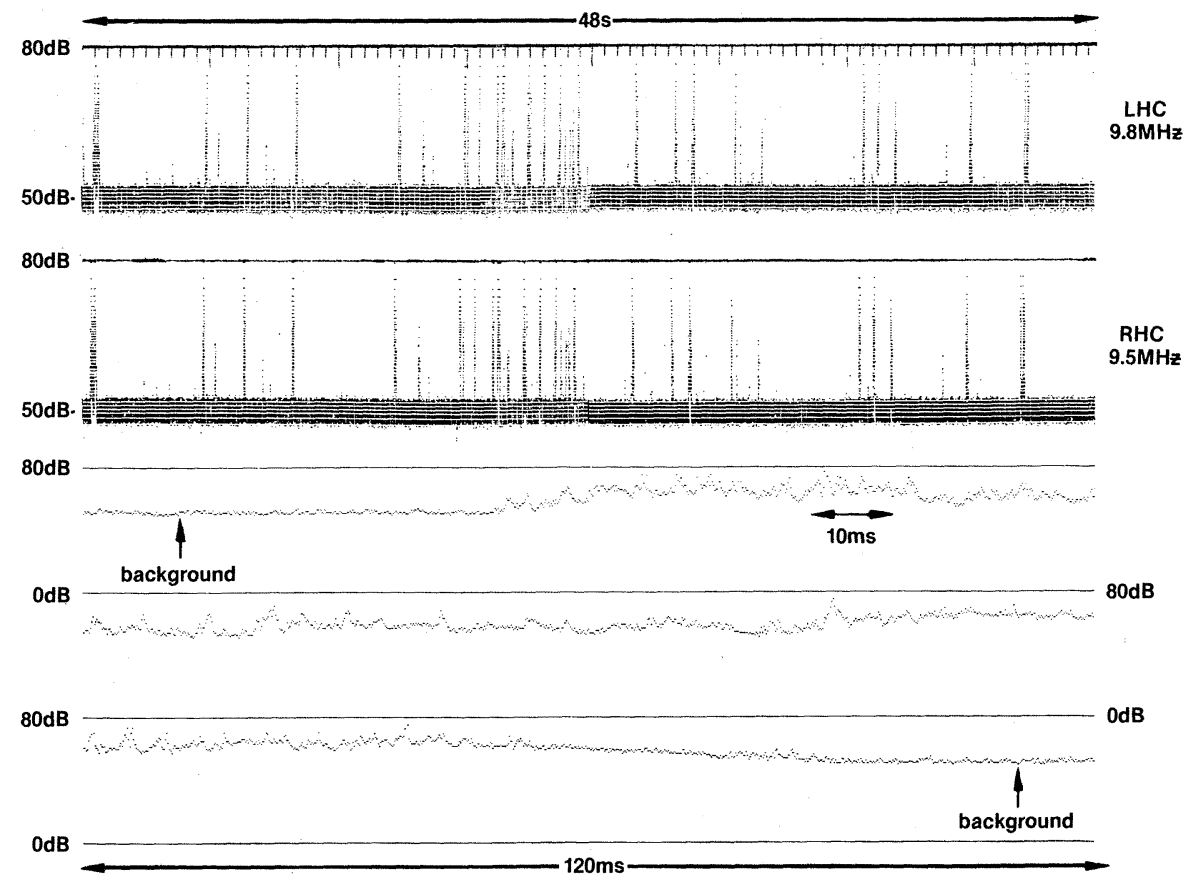
hours centered around closest approach. Figure 5 shows the occurrence of these events as a function of time and distance from Saturn. SED occur most frequently near closest approach and also in a series of episodes about 5 hours long each, during which the SED occurrence rate rises from an initially low level to a peak and then back down again. These episodes themselves repeat, with about five observable during the 48-hour interval centered near 0:00 hours on 13 November 1980 (spacecraft event time). The repetition period is about 11 hours. Since a rough 11-hour periodicity is present (consistent with the 10.657-hour Saturn rotation) and the occurrence rate of SED is inversely correlated with distance from Saturn, it is likely that the SED sources are near Saturn. Furthermore, several SED episodes were observed beyond the time span displayed in Fig. 5 and when Voyager was outside Saturn's magnetosphere. They could not have resulted from the interaction of Voyager with the plasma or particulate environment of Saturn.

Figure 6 shows data from the two PRA high-rate frames recorded nearest closest approach. Two more such frames 24 hours earlier and two 24 hours later still show SED, but weakly. SED occur simultaneously on channels spaced 307.2 $\mathrm{kHz}$ apart. If SED drifted in frequency (like Jupiter's millisecond bursts), we would probably see systematic differences between the two channels. Any drifts would have to be faster than $\simeq 10^{4}$ $\mathrm{MHz} \mathrm{sec}^{-1}$, which is three orders of magnitude faster than the fastest drifts in Jupiter's emission. Since the two channels measure opposite senses of polarization and the signals in the two channels are virtually identical, we conclude that SED is unpolarized (6). Finally, the shortest time structures are of the order of $140 \mu \mathrm{sec}$, during which the flux density changes by a factor of 10 . Using the customary criterion that source size cannot exceed the light travel distance corresponding to the shortest pulse time scale, we deduce that the emission source is not larger than $140 \mu \mathrm{sec} \times$ $300,000 \mathrm{~km} \cdot \mathrm{sec}^{-1}=50 \mathrm{~km}$.

Figure 4 shows that SED can occur anywhere in the PRA spectrum, from 20 $\mathrm{kHz}$ to $40.2 \mathrm{MHz}$. Our interpretation is that we are observing broadband bursts lasting typically a few tens of milliseconds but continuing sometimes as long as several hundred milliseconds. These bursts are observed in whichever PRA channel the receiver happens to be tuned when the SED takes place. Considering the lack of frequency drifts, the lack of a polarization signature, and the randomness of occurrence throughout the PRA spectrum, we believe that the simplest interpretation of SED is that it is a broadband (at least 40-MHz) phenomenon, identical in all these respects to the electromagnetic emissions of terrestrial lightning when it is observed at high and very high frequencies (7).

The fact that SED appears even in the PRA lowband data below $100 \mathrm{kHz}$ (Fig. 4) demonstrates that there is no ionosphere between Voyager 1 and the SED source. The peak Saturn electron density observed by the Voyager radio science team corresponded to a cutoff frequency of $1.370 \mathrm{MHz}(8)$. This peak, of course, occurs at a level far above Saturn's clouds. Therefore, the source of SED probably lies outside Saturn's atmosphere. Its source clearly is not in the electrical discharges that might be expected to occur in cumulonimbus clouds over Saturn.

If we assume that SED are isotropic in their radiation pattern and uniform in flux density over $100 \mathrm{MHz}$ of spectrum, then their total radiated power is $10^{7}$ to $10^{8} \mathrm{~W}$. Viewed as a whole, Saturn emits these bursts about 1 percent of the time; the average SED power is therefore $10^{5}$ to $10^{6} \mathrm{~W}(9)$.

The plasma environments of neither Jupiter nor Earth produce phenomena such as SED. The obvious distinction of Saturn lies in its well-developed ring system. We believe, therefore, that the most probable source of SED is Saturn's rings. Voyager 2 will fly past Saturn closer to the ring system than did Voyager 1 and should be able to test this conclusion. Terrestrial thunderstorms appear to involve dielectric particles, violent aerodynamic shearing motion, and an electric potential gradient (10). Saturn's rings certainly contain dielectric particles. The different orbital velocities of the Keplerian particles making up the rings constitute a strongly sheared motion, in which any particle is always overtaking another and in turn being overtaken. In one revolution a given particle of the B ring has a strong chance of hitting at least one other particle; the impact provides a rapid exchange of charge between particles. Finally, the rings possess a very dilute atmosphere of hydrogen evaporated from the particles themselves (11). If we suppose corotation of the magnetic field lines of force throughout that region, then the differential motion of ring particles and magnetic field will generate electric fields in the rings. From the corotation point at $\sim 1.87 R_{\mathrm{S}}$ near the outer edge of the $\mathrm{B}$ ring, the fields are inward inside $1.87 R_{\mathrm{S}}$ and outward outside. The existence of a corotating magnetic field is strongly suggested by the fact that SED appears in episodes, with a periodicity of $\sim 11$ hours.

The localization to the vicinity of Saturn, the geometric dimensions, and the physical nature of SED suggest that electrostatic phenomena play a strong role in the fine structure of Saturn's rings. The appearance in SED of modulation controlled by solar time and the rotation of Saturn suggests that sunlit and shadowed portions of the rings play contrasting roles in the generation of electrostatic phenomena. It remains a mystery to us why the magnetic field of Saturn should manifest its asymmetries even in the rings.

J. W. WARWICK

J. B. Pearce, D. R. Evans

Radiophysics, Inc.,

Boulder, Colorado 80301

T. D. CARr, J. J. Schauble

Department of Astronomy, University

of Florida, Gainesville 32611

J. K. Alexander

M. L. KaIser, M. D. DesCH

Laboratory for Extraterrestrial Physics, Goddard Space Flight Center,

Greenbelt, Maryland 20711

M. PEDERSEN

A. Lecacheux, G. Daigne

A. BoIschot, C. H. BARROW

Observatoire de Paris,

Section d'Astrophysique de Meudon, 92190 Meudon, France

References and Notes

1. M. L. Kaiser, M. D. Desch, J. W. Warwick, J. B. Pearce, Science 209, 1238 (1980).

2. In analogy to Jupiter's System III longitude convention, we have adopted a Saturn longitude system (SLS) based on a sidereal rotation period of 10 hours 39.4 minutes and an origin of longitude (SLS $=0^{\circ}$ ) toward Saturn's vernal equinox on 1.0 January 1980. When we use SLS we mean subspacecraft longitude, except where otherwise noted. See M. D. Desch and M. L. Kaiser (Geophys. Res. Lett., in press) for deKais
tails.
J. W

3. J. W. Warwick, J. B. Pearce, R. G. Peltzer, A. C. Riddle, Space Sci. Rev. 21, 309 (1977).

4. J. W. Warwick et al., Science 206, 991 (1979); A. Boischot et al., J. Geophys. Res., in press.

5. D. A. Gurnett et al., Science 212, 235 (1981).

. Episodes of SED after closest approach to Sat urn often do show polarization. We believe that this results from a propagation effect near Saturn as the planet limb occults the SED sources in the rings

7. J. W. Warwick, C. O. Hayenga, J. W. Brosnahan, J. Geophys. Res. 84, 2457 (1979).

8. G. L. Tyler et al., Science 212, 201 (1981).

9. At the distance of Earth this power corresponds to a flux density of about $0.1 \mathrm{Jy}\left(10^{-27} \mathrm{~W} \mathrm{~m} \mathrm{~m}^{-2}\right.$ $\mathrm{Hz}^{-1}$ ), which is probably undetectable in high and very. high frequencies. During SED events and very high frequencies. During SED events the flux density may. rise to 10 Jy and

10. R. Markson and M. Muire, Science 208, 979

$\rightarrow$ D. L. Judge, F.-M. Wu, R. W. Carlson, ibid. 207, 431 (1980).

12. We thank the radio science team for the use of their data prior to its publication, the other members of the planetary radio astronomy team for their support throughout the Voyager missions, and R. L. Poynter, M. Corn, R. Elson, and $B$. Razzaghinejad. The Voyager 1 PRA receiver, built by Martin-Marietta Corp., continues to operate flawlessly after nearly 4 years. Our French investigators acknowledge support by the Centre National d'Etudes Spatiales. This work is supported in part by JPL under NASA contract NAS 7-100.

9 February 1981 